\title{
Mechanical Eye Trauma Epidemiology, Prognostic Factors, and Management Controversies-An Update
}

\author{
Sharah Rahman 1* (D), Ava Hossain², Sarwar Alam³, Anisur Rahman4, Chandana Sultana3, \\ Saiful Islam ${ }^{3}$, Yusuf Jamal Khan'3, Md. Amiruzzaman'1 \\ ${ }^{1}$ Bangladesh Eye Hospital and Institute, Dhaka, Bangladesh \\ ${ }^{2}$ OSB Eye Hospital, Dhaka, Bangladesh \\ ${ }^{3}$ Ispahani Islamia Eye Institute and Hospital, Dhaka, Bangladesh \\ ${ }^{4}$ Dhaka Medical College and Hospital, Dhaka, Bangladesh \\ Email: ${ }^{\star}$ dr.sharahrahman@gmail.com
}

How to cite this paper: Rahman, S., Hossain, A., Alam, S., Rahman, A., Sultana, C., Islam, S., Khan, Y.J. and Amiruzzaman, Md. (2021) Mechanical Eye Trauma Epidemiology, Prognostic Factors, and Management Controversies-An Update. Open Journal of Ophthalmology, 11, 348-363. https://doi.org/10.4236/ojoph.2021.114029

Received: October 24, 2021

Accepted: November 27, 2021

Published: November 30, 2021

Copyright $\odot 2021$ by author(s) and Scientific Research Publishing Inc. This work is licensed under the Creative Commons Attribution International License (CC BY 4.0).

http://creativecommons.org/licenses/by/4.0/

\begin{abstract}
Purpose of Review: The management of eye injuries is both difficult and argumentative. This study attempts to highlight the management of ocular trauma using currently available information in the literature and author experience. This review presents a workable framework from the first presentation, epidemiology, classification, investigations, management principles, complications, prognostic factors, final visual outcome and management debates. Review Findings: Mechanical ocular trauma is a leading cause of monocular blindness and possible handicap worldwide. Among several classification systems, the most widely accepted is Birmingham Eye Trauma Terminology (BETT). Mechanical ocular trauma is a topic of unsolved controversy. Patching for corneal abrasion, paracentesis for hyphema, the timing of cataract surgery and intraocular lens implantation are all issues in anterior segment injuries. Regarding posterior segment controversies, the timing of vitrectomy, use of prophylactic cryotherapy, the necessity of intravitreal antibiotics in the absence of infection, the use of vitrectomy vs vitreous tap in traumatic endophthalmitis is the issues. The pediatric age group needs to be approached by a different protocol due to the risk of amblyopia, intraocular inflammation, and significant vitreoretinal adhesions. The various prognostic factors have a role in the final visual outcome. B scan is used to exclude R.D, Intraocular foreign body (IOFB), and vitreous haemorrhage in hazy media. Individual surgical strategies are used for every patient according to the classification and extent of the injuries. Conclusion: This article examines relevant evidence on the management challenges and controversies of mechanical trauma of the eye
\end{abstract}


and offers treatment recommendations based on published research and the authors' own experience.

\section{Keywords}

Mechanical Eye Trauma, Bermingham Eye Trauma Terminology, Prognostic Factors for Mechanical Trauma, Epidemiology of Mechanical Eye Injury, Open Globe Injuries (OGI), Ocular Trauma Scoring (OTS), Classification and Regression Tree (CART) Model, Update of Mechanical Eye Trauma, Classification of Ocular Trauma, Controversies of Ocular Trauma, Challenges in Ocular Trauma Management

\section{Introduction}

Ocular trauma is a common, preventable cause of visual impairment. From developing to developed countries, eye injury causes a social burden to the community. The treating of eye injuries is both problematic and debatable [1]. There are controversies regarding classification system, treatment plan, management complication, need for multiple surgeries etc. when it comes to ocular trauma. The Beaver Dam Eye Study finds that the lifetime frequency of ocular trauma is 14.4 - 19.8 per cent [2]. Mechanical eye injuries affect the eye in many ways ranging from mild to severe morbidity. The burden of blindness is related to both its unavoidable impact on quality of life and the subsequent loss of production in these persons. Ocular trauma affects up to 6 million children worldwide each year, with a quarter-million requiring hospitalization [3]. The causative factors are widely variable and have a direct impact on visual outcome. Several questions like cause of the trauma, extent of the trauma, initial visual acuity, time of starting treatment, need of multiple surgery, association of endophthalmitis, counselling and realistic outcome of the management will be discussed. The management of ocular injuries in the paediatric age group differs significantly from adults. Over the last 30 years, developments in our understanding of the pathogenesis and management of ocular trauma, together with advances in ocular surgery apparatus and procedures, have enhanced the success of the final visual outcome. Mechanical eye injuries have a deep impact on the patient's life as well as to the family and society. But it can be avoidable with social awareness and some protective measure. This review article will focus on the measure, that should be taken to avoid mechanical eye trauma, and we believe it will give the trauma surgeons overall guidance.

\section{Methods of Literature Search}

The following flow chart in Figure 1 explains the literature review process.

\section{Epidemiology of Ocular Trauma}

There is a wide range of demographic variations in the epidemiology of ocular 


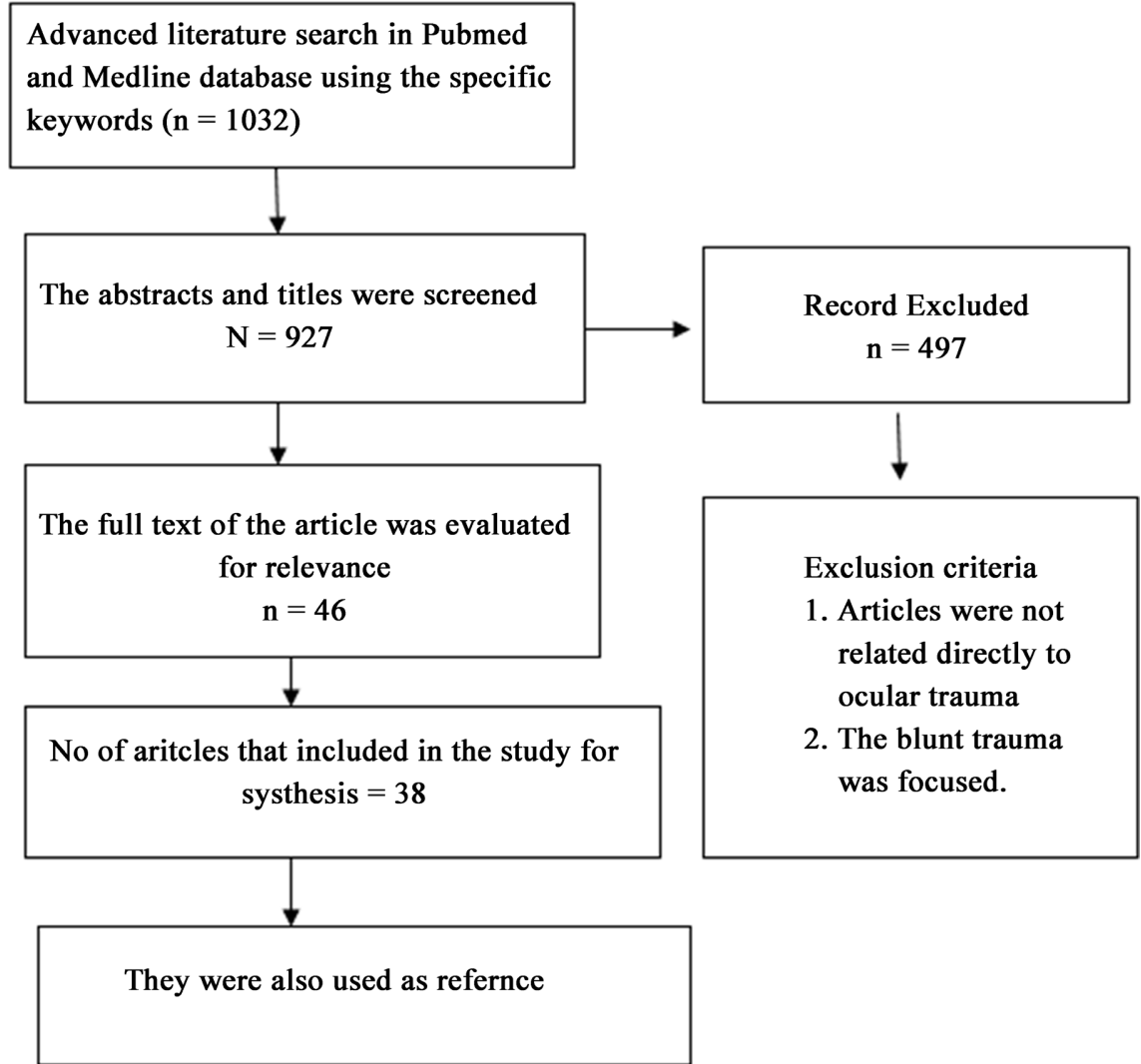

Figure 1. Literature review process.

trauma. The mean age is in between the second to the fourth decade [4] [5]. The highest rate of eye trauma is between 15 - 20 years. (11.5 per cent) [6]. Males were more prone to injuries than females mentioned by different authors $84.6 \%$ [5], 74\% [6] and $87.6 \%$ [7]. It is a primary cause of monocular blindness, accounting for approximately $60 \%$ of cases, particularly among children. [8] [9] [10]. Open globe injuries (OGI) are more common than closed globe injuries (CGI), with a proportion of approximately $60 \%$ vs $40 \%$ [3] [11] [12]. Sharp items were responsible for more than half of all injuries. The most prevalent cause was work-related injuries, followed by falls [10]. For children, the most prevalent location of the injury is the home [13]. Penetrating corneal injury was the most common type among open globe injuries, followed by rupture globe, perforation, and Intra-ocular foreign body [11] [14]. Among the closed globe injuries, contusion was most common, followed by laceration [14] [15].

\section{Clinical Classification of Mechanical Trauma of the Eye}

Open globe injury (OGI) and closed globe injury (CGI) are two types of mechanical eye injuries (CGI). Birmingham Eye Trauma Terminology (BETT) is a widely used ocular trauma terminology system. It provides a precise definition 
for each injury type and a comprehensive framework for the injury type (Table 1 \& Box 1). Although BETT exclusively addresses mechanical ocular injuries [15] in terms of OGI, but avoids any ambiguity in communication. As a result, BETT cannot classify orbital and ocular adnexa injuries, and various categorization systems have been proposed [15]. Grant and colleagues demonstrated the importance of lid and adnexal laceration in predicting the ultimate visual outcome in the Classification and Regression Tree analysis model (CART) [16]. Agrawal et al. [16] also recommended to include orbital and adnexal trauma. They suggested that anatomical landmarks such as the spiral of Tillux or the annulus of Zinn can be used to separate two zones and be included in future prognostic models [1] [17].

Table 1. The terminology used in BETT.

\begin{tabular}{cl}
\hline BETT & Birmingham Eye Trauma Terminology \\
OGI & Open globe injury \\
Closed globe injury \\
Penetrating & Full-thickness corneoscleral wound with no exit wound \\
Perforating & Full-thickness corneoscleral wound with an exit wound \\
IOFB & Intraocular foreign body retained in the globe \\
\hline
\end{tabular}

Type:
A. Rupture
B. Penetrating
C. Intraocular foreign body
D. Perforating
E. Mixed
Grade:
Visual activity
1. $\$ 20 / 40$
2. $20 / 50$ to $20 / 100$
3. $19 / 100$ to $5 / 200$
4. $4 / 200$ to light perception
5. No light perception

Pupil

1. Positive: relative afferent pupillary defect present in affected eye

2. Negative: relative afferent pupillary defect absent in affected eye

Zone

I. Isolated to cornea (including the corneo-scleral limbus)

II. Corneo-scleral limbus to a point $5 \mathrm{~mm}$ posterior into the sclera

III. Posterior to the anterior $5 \mathrm{~mm}$ of sclera

Box 1. Open globe injury classification. 


\section{Causology}

There are several causative factors responsible for mechanical eye trauma. Among them, road traffic accident (RTA) is commonest in adult [18]. Sportsrelated injury is more common in children [19]. Other common causes are occupational injury, chemical burn, bomb blast, particle related injury, especially iron particle, blow to the eye like stone, fist or stick.

\section{Prognostic Indicators for Ultimate Visual Outcome}

The CART (Figure 2) [15] [16] and the ocular trauma score (OTS) (Table 2)

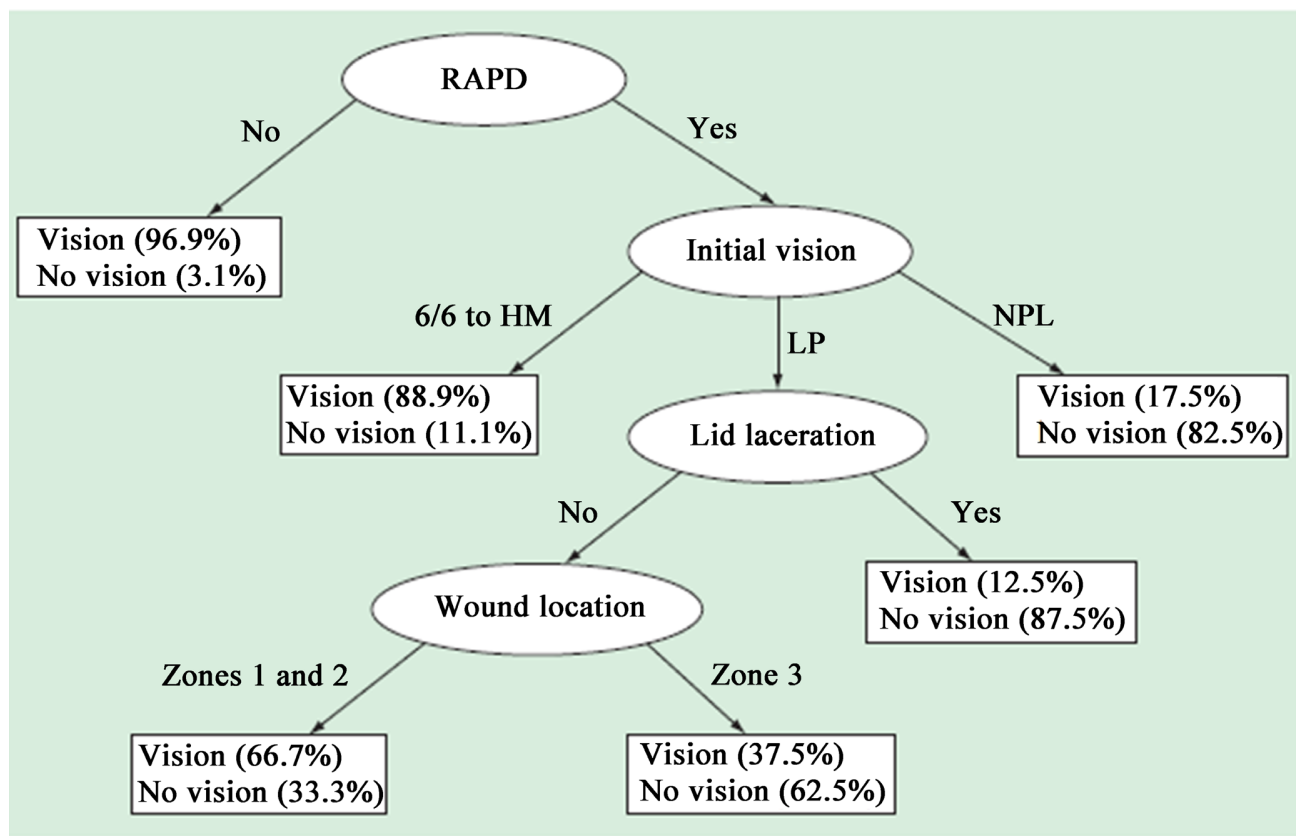

Figure 2. Classification and regression tree model for open globe injury: visual survival (light perception or better) vs no visual survival. H.M.: Hand moment; L.P.: Light perception; NPL: No light perception; RAPD: Relative afferent pupillary defect.

Table 2. Summation of raw points of ocular trauma score (OTS).

\begin{tabular}{llr}
\hline 1. Initial visual acuity (without correction) & $\begin{array}{l}\text { No light perception } \\
\text { Light perception/hand movement }\end{array}$ & 70 \\
& $1 / 200$ to $19 / 200$ & 80 \\
& $20 / 200$ to $20 / 50$ & 90 \\
& $>20 / 40$ & 100 \\
& & -23 \\
2. Rupture globe & & -17 \\
3. Endophthalmitis & & -14 \\
4. Perforating ocular injury & -11 \\
5. Retinal detachment (RD) & -10 \\
6. Afferent pupillary defect (RAPD) & &
\end{tabular}


[18] are two techniques for determining future visual prognosis following OGI.

The Ocular Trauma Classification Group defines wound location [20]. The OTS is computed by allocating numerical raw points to six variables: initial vision, an RAPD, perforating injury, rupture globe, endophthalmitis, and retinal detachment. These six prognostic factors are significantly related to the final visual outcome. The ratings are divided into five groups based on the likelihood of achieving a range of post-injury visual acuities (Table 2). The higher the OTS score, the more favourable the prognosis. The existence of an RAPD and poor initial visual acuity is the most predictive of vision loss in the CART classification tree; the presence of a laceration on the lid and the posterior position of the wound further predicted a poor visual outcome. According to Wai and Steel [19], while comparing OTS and CART models in the Management of OGI, the OTS had superior predictive accuracy. OTS is a helpful tool for patient counselling and care [21]. OTS has been verified in various series [16] and provides trauma patients and families with evidence-based predictive outcomes. On the U.S. Eye Injury website, one may also compute the OTS score [22].

\section{Initial Evaluation of Ocular Trauma}

\subsection{History}

The processes, timing, and circumstances surrounding the injury should be meticulously documented, as they may become essential pieces of medicolegal evidence in the future. Any history of treatment, including medicine, surgery, or amblyopia, should be recorded. Type, place, timing, causative factors of the injury should be evaluated.

\subsection{Systemic Evaluation}

Any life-threatening damage should be ruled out before approaching any patient with ocular trauma in emergency settings. Basic and advanced life support are the primary goals in severe injuries until the patient's stabilization. Any defect in the pupil needs a complete evaluation of the posterior segment, including the retina, choroid, and optic nerve. Eyeshield should be placed over the eye immediately, and any pressure or eye drop should be avoided. Tetanus toxoid is recommended to prevent tetanus.

\subsection{Assessment of Vision}

The visual status of the patient should be recorded in each eye by a standard vision chart. Any counting finger, hand movement, accurate projection in all quadrants, and perception of light (P.L.) should be appropriately recorded. The absence of light perception is not an indication for enucleation, and the globe may be salvageable [23].

\subsection{Preoperative Ophthalmic Evaluation}

Firstly, a thorough assessment of the mechanical injury is done. The primary 
management needs a complete ophthalmic examination, emphasizing the possibility of eye penetration and incorporating gonioscopy and dilated fundoscopy [24]. If the study of the globe is challenging, computed tomography (C.T) can help. Due to management and medicolegal considerations, it is critical to record visual acuity as the initial step in approaching a patient with ocular damage. In all situations, photographic evidence or a diagrammatic depiction of the kind and degree of the damage should be made (Figure 3). Mechanical OGI should be classified according to the international classification system [20] to prevent further ambiguity among the team. An anaesthetic examination is recommended to rule out globe disintegrate when OGI is suspected, but a thorough examination is not feasible (e.g., pediatric trauma).

\section{Ophthalmic Manifestations of Ocular Trauma}

1) Eye Lid and Adnexa: Edema, ecchymosis, and panda eyes, laceration, canaliculi injury and lid margin involvement

2) Extraocular muscle: Ocular motility should be tested in all positions of gaze in suspected orbital or cranial nerve injury with caution.

3) Orbit: Proptosis or enophthalmos. Crepitus on palpation, discomfort on palpation of bony orbital edge to rule out an orbital fracture. In pediatric orbital trauma, white eye orbital fracture should be checked carefully.

4) Conjunctiva: Injection, chemosis, haemorrhage, emphysema, pigmentation suggestive of uveal tissue involvement.

5) Cornea: Penetrating corneal injury with an extension of the wound, iris/ uveal prolapse, IOFB. In suspected self-sealing wounds, Siedel's test is recommended.

6) Limbus: It acts as a landmark. Limbus can be involved in the lacerated injury of cornea, or sclera or sclera-corneal injury as well.

7) Sclera: Rupture globe or penetrating scleral injury with or without posterior extension, prolapse of intraocular content like choroid, vitreous, retinal etc.

8) Anterior chamber (A.C.): Depth of A.C., cells, hyphema, hypopyon, fibrin, membrane, lens particle, vitreous, IOFB.

9) Iris: Sphincter tear, iridodialysis, iris prolapse with loss of viability, aniridia,

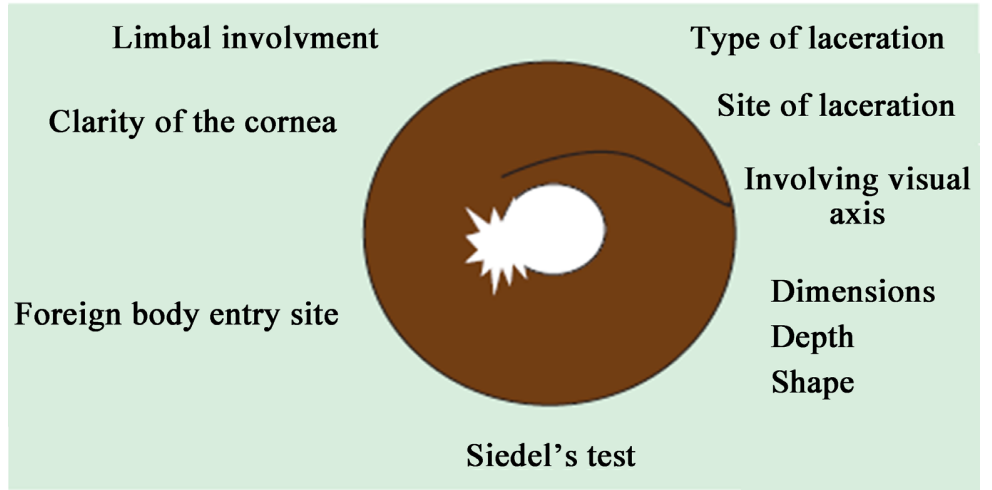

Figure 3. Pictorial demonstration of corneal injury. 
angle recession, cyclodialysis cleft, iritis, iris atrophy, short miosis, traumatic dilatation of the pupil.

10) Crystalline Lens: Traumatic cataract, anterior capsule injury, posterior capsule rupture (PCR), zonular dehiscence, subluxation, dislocation, Vossius ring.

11) Vitreous: Haemorrhage, posterior vitreous detachment, IOFB

12) Retina: Retinal tear, subhyaloid or intraretinal haemorrhage, IOFB, retinal detachment, choroidal detachment, choroidal rupture, macular hole, Berlin's oedema, traumatic endophthalmitis.

13) Intraocular pressure (IOP): IOP should be checked by non-contact tonometry in all cases if the patient allows it. It should not, however, be done if a globe rupture is suspected

14) Gonioscopy: In all trauma cases, a gonioscopy should be performed, especially following the absorption of hyphema, to check any angle recession or IOFB or pigmentation. It should not be attempted in cases when OGI is suspected.

\section{Role of Imaging in the Management of Ocular Trauma}

Radiography, ultrasonography (USG), MRI, and C.T. are all widely used imaging technologies for the orbit and eye. In cloudy media, B-scan USG can be highly effective. In OGI, a gentle B Scan is recommended. C.T. Scan is commonly used in pinpointing the exact location of an IOFB or orbital damage. Zhang et al. [25] reported that C.T. could fail to detect metal fragments $<0.5 \mathrm{~mm}$. If a metallic intraocular or intraorbital foreign body is suspected, an MRI is not recommended.

\section{The Management Approach for Mechanical Eye Trauma}

The fundamental goal of primary globe repair is to restore anatomical integrity while optimizing visual potential.

The four-pronged method for open-world injury management should include general principles for risk avoidance and outcome optimization.

1) Reduce the risk of further trauma;

2) Reduce the risk of infection;

3) Reduce the trauma victim's and his family's psychological trauma and

4) Control the legal problems.

\section{Preoperative Planning}

\subsection{Surgery Timetable}

Unless prohibited by any other medical condition, primary globe repair should be performed as soon as possible, preferably within the first 24 hours.

\subsection{Consent and Counseling}

The ophthalmologist must adequately inform the patient and family about the 
status of the eye, the surgical plan, costing and its complications. This should include that multiple surgeries may be needed, and primary repair is only the first step. The patient should be told of the OGI's guarded prognosis before surgery to ensure reasonable expectations.

\subsection{Anaesthesia}

Although the choice of anaesthetic medication, particularly in OGI, is debatable, general anaesthesia is advised for ocular injuries. We have performed repair under peribulbar anaesthesia in our unpublished data, with an excellent postoperative outcome (Figure 4).

\section{Surgical Approach of Mechanical Trauma}

\subsection{Exposure of the Eye}

The eye should be adequately exposed. If lid damage or a periorbital hematoma is hampering the view, it should be addressed initially.

\subsection{Cornea \& Sclera}

Foreign debris and membrane should be removed during the initial exploratory operation. 360 degree conjunctival peritomy is ideal. Peroperative assessment of the injury with extension and meticulous planning is essential. Limbus should be secured first, followed by sclera and cornea. Usually, 10-0 monofilament Nylon is used for cornea, 8-0 Vicryl for sclera and 8-0 silk for conjunctiva. Corneal sutures should be placed radially and neither be too tight to distort the cornea nor too loose, leading to wound dehiscence. Corneal sutures should be shorter in the centre of the cornea than in the peripheral cornea (Figure 5). Experienced surgeons should perform the repair to reduce the mal handling of the delicate cornea and optimize the outcome. Stellate corneal laceration should be repaired using purse-string sutures.

Scleral wounds that extend beyond the rectus insertion are healed as far back

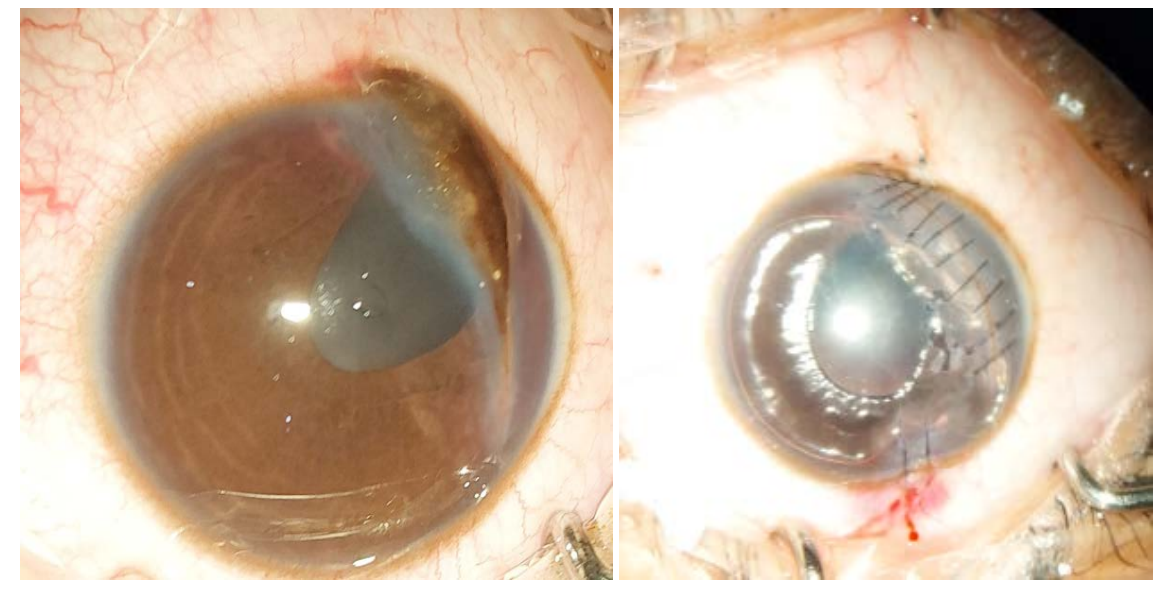

Figure 4. Preoperative and Postoperative picture of penetrating corneal injury repair performed under local anaesthesia. 


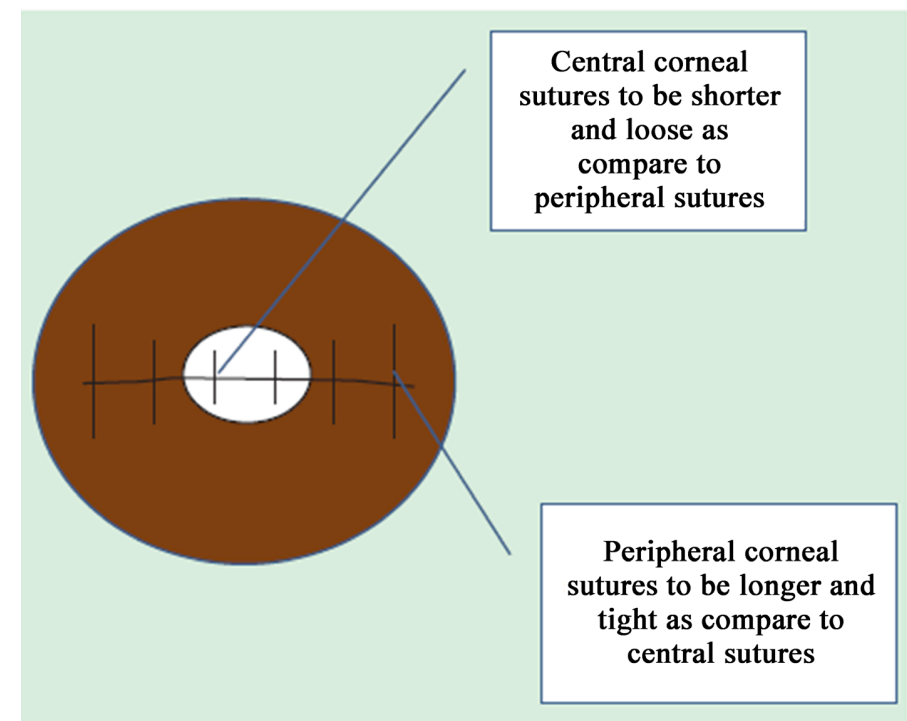

Figure 5. Diagram of corneal laceration repair with different suture lengths in the centre and mid-periphery.

as feasible. The margin should be free of any uveal tissue. Any prolapsing vitreous should be cleared by anterior vitrectomy to avoid traction on the vitreous and retina.

\subsection{Iris}

If the iris is viable, should try to preserve it as much as possible. In lacerated iris, pupilloplasty may be needed. In the case of irido-dialysis, a repair can be done by swing technique or modified McCalan suture.

\subsection{Lens}

Traumatic cataracts can develop due to a breach in the capsule or perforating injury. Zonular dehiscence, anterior and posterior capsular tear, subluxation or dislocation may further compromise the lens status. Cataract extraction with simultaneous repair of the penetrating injury and IOL implantation has been widely recommended [26]. IOL may be inserted in the first sitting, or secondary IOL can be given later on. Biometry of the better eye is widely established in case traumatic eye [27]. Postoperative visual outcome following traumatic cataract correction revealed $31 \%$ of eyes had a visual acuity $>20 / 40$, and 61.0 per cent had a visual acuity $>20 / 200$, according to Shah et al. [10] [26]. The secondary IOL implantation and primary IOL implantation shoes similar visual outcomes [28], though secondary IOL implantation allows the affected eye time to heal and the surgeon more time to plan. A separate incision site is recommended for cataract extraction, and cataracts should never be extracted through the site of laceration.

\subsection{Vitrectomy}

Vitreoretinal surgical techniques allow many poor prognosis eyes to be salvaged [29]. Broadly, indications are R.D. with or without proliferative retinopathy 
(PVR), IOFB, lens-related problems and others. Scleral buckling also can be considered in traumatic R.D. without vitreous haemorrhage or PVR.

\subsection{Intraocular Foreign Body}

IOFB injuries are relatively complex eye emergencies, necessitating comprehensive treatments and often serial surgeries [30]. They cause mechanical injury along the track, carry infections, increasing the risk of endophthalmitis, toxic chemical reactions by the retained IOFB, especially with metal IOFBs. Early removal of IOFB is optimal to decrease the risk of endophthalmitis and PVR [31]. However, in a study by Colyer et al. [32], 54.0\% of patients achieved good visual outcomes when the IOFB was removed after a median of 21 days after providing adequate cover with a combination of systemic and topical antibiotics.

\subsection{Retinal Detachment}

Traumatic R.D may occur due to retinal dialysis, flap tears, giant retinal tears, retinal necrosis. Eyes with trauma and retinal tear are at high risk of PVR and likely to undergo vitrectomy. It can be present at the time of injury or develop post-globe repair, and the incidence varies from $2.5 \%$ to $39 \%$ [33]. Longer wound length $(>10 \mathrm{~mm})$, vitreous prolapse, vitreous haemorrhage, rupture injury (vs laceration) and retained IOFB are other factors that cause retinal detachment.

\subsection{Traumatic Hyphema}

Traumatic hyphema involving more than half of the cornea may cause permanent vision loss due to blood-stained cornea or secondary glaucoma causing optic neuropathy. Reactionary bleeding could lead to permanent vision impairment, especially in patients with sickle cell trait/disease. Walton et al. [34] recommended routine use of topical cycloplegics and corticosteroids, systemic antifibrinolytic agents or corticosteroids and a rigid shield. They also recommend activity restriction (quiet ambulation) and interdiction of non-steroidal anti-inflammatory agents. The presence of corneal blood staining or severely elevated IOP after maximal tolerable medicinal treatment are indications for surgery.

\subsection{Traumatic Optic Neuropathy}

The optic nerve can be compromised either by direct compression due to inflammatory exudate and volume overload with the orbit or axonal swelling posttraumatic inflammation. Another mechanism can be the direct impingement of bony fragments within the optic canal. High dose steroids or orbital decompression is controversial in improving recovery compared with observation, and there are high rates of spontaneous recovery [35].

\section{Management of Infection/Inflammation}

\subsection{Endophthalmitis}

Post-traumatic endophthalmitis comprises $25 \%$ - $30 \%$ of all endophthalmitis cas- 
es, and it occurs in $3 \%-10 \%$ [36] of patients after penetrating injury. This rate changes to $6 \%-30 \%$ with the presence of an IOFB [37]. Early surgical repair and prophylactic systemic intravenous antibiotics for $48 \mathrm{~h}$ may reduce this incidence to $<1 \%$ [32]. Risk factors include iron-related injuries, severely lacerated eye, retained IOFB, lens capsule injury, and delayed repair. The treatment comprises prompt recognition, intervention such as vitreous tap or vitrectomy followed by intravitreal injection of antibiotics (e.g., vancomycin plus ceftazidime) and systemic therapy [36]. There is a high incidence of retinal detachment (17\% - 58\%) and risk of phthisis in eyes with post-traumatic endophthalmitis.

\subsection{Sympathetic Ophthalmia (SO)}

The time from ocular injury to onset of SO varies greatly, ranging from a few days to decades. $80 \%$ of the cases occurred within three months after damage to the exciting eye and $90 \%$ within one year [38]. Cyclosporine, chlorambucil, cyclophosphamide or azathioprine may also be utilized if the inflammatory reaction cannot be adequately controlled with corticosteroids alone.

\section{Postoperative Approach \& Follow-Up}

Postoperatively, a multidisciplinary approach to management and follow-up is essential for proper surgical rehabilitation of OGI patients [39]. A meticulous follow-up and documentation are necessary for the management of eyes with trauma. Recording of IOP, retina status and lens status and corneal sutures monitoring are all critical components in postoperative care of traumatized globe.

\section{Medicolegal Issue}

All the clinical and relevant findings and hospital visits should be well documented, timed and dated. This information may stand as substantial evidence in a court of law and in legal cases where compensations are granted against employers, other persons, etc. Proper documentation and decision were taken with the patient's involvement in general to minimize the risk of future litigations against treating physicians.

\section{Controversies Regarding the Management of Ocular Trauma}

Ocular trauma is a matter of unsolved controversies, and management techniques for open-globe injuries are constantly changing. The international classification of ocular trauma is old and should be revised to calculate the outcome more precisely. Ocular adnexa and orbit should be involved in the classification. Covering the eye for corneal abrasion, corneal laceration repair, and therapeutic management of hyphema are all issues in anterior segment trauma. Cataract extraction and intraocular lens implantation in the aftermath of trauma are still being argued across the world.

Regarding posterior segment controversies, early versus late vitrectomy, preo- 
perative application of cryotherapy and a scleral buckle, use of intravitreal antibiotic irrespective of infection, the utility of vitrectomy vs vitreous tap in the case of traumatic endophthalmitis are the issues. The significance of intravenous methylprednisolone in traumatic optic neuropathy is always debatable. The pediatric age group needs to be approached by a different protocol due to the risk of amblyopia, intraocular inflammation, and significant vitreoretinal adhesions. The discussion will give suggestions regarding the management of mechanical eye injuries based on existing published research and the writers' personal experience.

\section{Prevention and Protection of the Eye from Injury}

According to studies and research, 90\% of eye injuries are preventable [19]. Wearing safety glasses or goggles whenever you deal with chemicals or around the metal, glass, or other materials that could fly into your eyes is the safest way to safeguard your eyes. Squash and racquetball players must use eye protection. Protect your eyes with a shield affixed to your helmet if you play baseball or football. The children should be kept monitored. Sharp objects like pencils, pens, scissors should be kept away from the child. Because most eye injuries are preventable, emphasis needs to be placed on preventing rather than curing them. It is essential to raise public awareness about the benefits of wearing protective equipment, which is broadly accessible, which was the primary concern of most of the authors who reported on ocular trauma in the previous year.

\section{Key Summarization}

1) In ocular trauma, any life-threatening damage should be checked out first.

2) Determine visual acuity in both eyes with ocular injuries at the time of presentation.

3) Document all results legibly using clinical photos or diagrammatic depiction, as some instances may be medicolegal.

4) To avoid misunderstanding in communication, use the worldwide nomenclature and categorization system in ocular trauma eyes.

5) Because counselling is the mainstay of trauma patient care, physicians must provide patients with tools such as the ocular trauma score, which should be utilized regularly in conjunction with standardized nomenclature for ocular trauma.

6) In the management of complicated traumatized eyes, a devoted multidisciplinary team is required.

7) Globe injuries should be handled according to the broad principles of ocular trauma management, and surgical restoration of these eyes should follow the "atraumatic repair" approach.

\section{Conclusion}

Traumatic mechanical eye injuries can have substantial morphological and functional consequences on ocular tissue structures. Despite advances in diagnostic 
and surgical methods, globe rupture frequently results in vision loss. A thorough assessment and an appropriate technique are critical for successful treatment and preserving the final visual outcome.

\section{Acknowledgements}

We acknowledge Taskin Jamal, PhD. for his active support during the research.

\section{Conflicts of Interest}

The authors declare no conflicts of interest regarding the publication of this paper.

\section{References}

[1] Agrawal, R., et al. (2013) Controversies in Ocular Trauma Classification and Management: Review. International Ophthalmology, 33, 435-445.

https://doi.org/10.1007/s10792-012-9698-y

[2] Wong, T.Y., Klein, B.E. and Klein, R. (2000) The Prevalence and 5-Year Incidence of Ocular Trauma. The Beaver Dam Eye Study. Ophthalmology, 107, 2196-2202. https://doi.org/10.1016/S0161-6420(00)00390-0

[3] Barry, R.J., et al. (2019) The UK Paediatric Ocular Trauma Study 3 (POTS3): Clinical Features and Initial Management of Injuries. Clinical Ophthalmology, 13, 11651172. https://doi.org/10.2147/OPTH.S201900

[4] Ozturk, T., et al. (2019) Etiology and Visual Prognosis in Open Globe Injuries: Results of a Tertiary Referral Center in Turkey. Scientific Reports, 9, Article No. 17977. https://doi.org/10.1038/s41598-019-54598-w

[5] Cillino, S., et al. (2008) A Five-Year Retrospective Study of the Epidemiological Characteristics and Visual Outcomes of Patients Hospitalized for Ocular Trauma in a Mediterranean Area. BMC Ophthalmology, 8, 6.

https://doi.org/10.1186/1471-2415-8-6

[6] Pandita, A. and Merriman, M. (2012) Ocular Trauma Epidemiology: 10-Year Retrospective Study. New Zealand Medical Journal, 125, 61-69.

[7] Jafari, A.K., et al. (2010) Epidemiology and Sociodemographic Aspects of Ocular Traumatic Injuries in Iran. International Ophthalmology, 30, 691-696. https://doi.org/10.1007/s10792-010-9401-0

[8] Bandrakalli, P., et al. (2012) Prevalence and Causes of Monocular Childhood Blindness in a Rural Population in Southern India. Journal of Pediatric Ophthalmology \& Strabismus, 49, 303-307. https://doi.org/10.3928/01913913-20120515-03

[9] Kömpf, D., Hacke, W. and Hanley, D.F. (1994) Acute Visual Loss-Monocular Blindness. In: Hacke, W., et al., Eds., Neurocritical Care, Springer, Berlin, 344-352. https://doi.org/10.1007/978-3-642-87602-8 33

[10] Fujikawa, A., et al. (2018) Visual Outcomes and Prognostic Factors in Open-Globe Injuries. BMC Ophthalmology, 18, 138. https://doi.org/10.1186/s12886-018-0804-4

[11] Shah, S.M., et al. (2020) A Prospective Cohort Study on the Epidemiology of Ocular Trauma Associated with Closed-Globe Injuries in Pediatric Age Group. Indian Journal of Ophthalmology, 68, 500-503. https://doi.org/10.4103/ijo.IJO 46319

[12] Yang, G.-Q., Xiong, Q.-C. and Wang, L.-L. (2013) Clinical Characteristics, Diagnosis and Treatment of Mechanical Ocular Trauma in 122 Cases. International Eye Science, 13, 1912-1914. 
[13] Kadappu, S., Silveira, S. and Martin, F. (2013) Aetiology and Outcome of Open and Closed Globe Eye Injuries in Children. Clinical \& Experimental Ophthalmology, 41, 427-434. https://doi.org/10.1111/ceo.12034

[14] Singh, R., et al. (2003) Characterization and Causes of Open Globe Injuries among Different Age Groups Presenting to a Tertiary Care Facility. Investigative Ophthalmology \& Visual Science, 44, 784-784.

[15] Xiao, J.H., et al. (2014) A New Classification for Epidemiological Study of Mechanical Eye Injuries. Chinese Journal of Traumatology, 17, 35-37.

[16] Schmidt, G.W., et al. (2008) Vision Survival after Open Globe Injury Predicted by Classification and Regression Tree Analysis. Ophthalmology, 115, 202-209.

https://doi.org/10.1016/j.ophtha.2007.04.008

[17] Agrawal, R., Ho, S.W. and Teoh, S. (2013) Pre-Operative Variables Affecting Final Vision Outcome with a Critical Review of Ocular Trauma Classification for Posterior Open Globe (Zone III) Injury. Indian Journal of Ophthalmology, 61, 541-545. https://doi.org/10.4103/0301-4738.121066

[18] Ababneh, L.T., et al. (2019) Hospital-Based Ocular Trauma: Factors, Treatment, and Impact Outcome. Clinical Ophthalmology (Auckland, N.Z.), 13, 2119-2126. https://doi.org/10.2147/OPTH.S223379

[19] Wagner, P.J. and Lang, G.K. (1996) Mechanical Ocular Trauma. Current Opinion in Ophthalmology, 7, 57-64. https://doi.org/10.1097/00055735-199608000-00011

[20] Pieramici, D.J., et al. (1997) A System for Classifying Mechanical Injuries of the Eye (Globe). The Ocular Trauma Classification Group. American Journal of Ophthalmology, 123, 820-831. https://doi.org/10.1016/S0002-9394(14)71132-8

[21] Yu Wai Man, C. and Steel, D. (2010) Visual Outcome after Open Globe Injury: A Comparison of Two Prognostic Models-The Ocular Trauma Score and the Classification and Regression Tree. Eye (London), 24, 84-89.

https://doi.org/10.1038/eye.2009.16

[22] Kuhn, F., et al. (2006) Epidemiology of Blinding Trauma in the United States Eye Injury Registry. Ophthalmic Epidemiology, 13, 209-216. https://doi.org/10.1080/09286580600665886

[23] Agrawal, R., Wei, H.S. and Teoh, S. (2012) Predictive Factors for Final Outcome of Severely Traumatized Eyes with No Light Perception. BMC Ophthalmology, 12, 16. https://doi.org/10.1186/1471-2415-12-16

[24] Saeed, A., et al. (2008) Plain X-Ray and Computed Tomography of the Orbit in Cases and Suspected Cases of Intraocular Foreign Body. Eye (London), 22, 1373 1377. https://doi.org/10.1038/sj.eye.6702876

[25] Zhang, Y., et al. (2009) Tiny Ferromagnetic Intraocular Foreign Bodies Detected by Magnetic Resonance Imaging: A Report of Two Cases. Journal of Magnetic Resonance Imaging, 29, 704-707. https://doi.org/10.1002/jmri.21637

[26] Tyagi, A.K., et al. (1998) Simultaneous Posterior Chamber Intraocular Lens Implant Combined with Vitreoretinal Surgery for Intraocular Foreign Body Injuries. Eye (London), 12, 230-233. https://doi.org/10.1038/eye.1998.55

[27] Cohen, K.L. (2001) Inaccuracy of Intraocular Lens Power Calculation after Traumatic Corneal Laceration and Cataract. Journal of Cataract \& Refractive Surgery, 27, 1519-1522. https://doi.org/10.1016/S0886-3350(01)00872-0

[28] Chuang, L.H. and Lai, C.C. (2005) Secondary Intraocular Lens Implantation of Traumatic Cataract in Open-Globe Injury. The Canadian Journal of Ophthalmology, 40, 454-459. https://doi.org/10.1016/S0008-4182(05)80005-5 
[29] Mieler, W.F. and Mittra, R.A. (1997) The Role and Timing of Pars Plana Vitrectomy in Penetrating Ocular Trauma. Archives of Ophthalmology, 115, 1191-1192. https://doi.org/10.1001/archopht.1997.01100160361017

[30] Mester, V. and Kuhn, F. (2002) Intraocular Foreign Bodies. Ophthalmology Clinics of North America, 15, 235-242. https://doi.org/10.1016/S0896-1549(02)00013-5

[31] Greven, C.M., et al. (2000) Intraocular Foreign Bodies: Management, Prognostic Factors, and Visual Outcomes. Ophthalmology, 107, 608-612. https://doi.org/10.1016/S0161-6420(99)00134-7

[32] Colyer, M.H., et al. (2007) Delayed Intraocular Foreign Body Removal without Endophthalmitis during Operations Iraqi Freedom and Enduring Freedom. Ophthalmology, 114, 1439-1447. https://doi.org/10.1016/j.ophtha.2006.10.052

[33] Lai, W.Y. and Wu, T.T. (2016) Successful Management in a Case of Traumatic Retinal Detachment Due to Open Globe Injury Using Microincisional Vitrectomy. Case Reports in Ophthalmology, 7, 476-480. https://doi.org/10.1159/000450638

[34] Walton, W., et al. (2002) Management of Traumatic Hyphema. Survey of Ophthalmology, 47, 297-334. https://doi.org/10.1016/S0039-6257(02)00317-X

[35] Yu-Wai-Man, P. and Griffiths, P.G. (2005) Surgery for Traumatic Optic Neuropathy. Cochrane Database of Systematic Reviews, No. 4, Cd005024. https://doi.org/10.1002/14651858.CD005024.pub2

[36] Andreoli, C.M., et al. (2009) Low Rate of Endophthalmitis in a Large Series of Open Globe Injuries. American Journal of Ophthalmology, 147, 601-608.e2. https://doi.org/10.1016/j.ajo.2008.10.023

[37] Lieb, D.F., et al. (2003) Open Globe Injuries with Positive Intraocular Cultures: Factors Influencing Final Visual Acuity Outcomes. Ophthalmology, 110, 1560-1566. https://doi.org/10.1016/S0161-6420(03)00497-4

[38] Goto, H. and Rao, N.A. (1990) Sympathetic Ophthalmia and Vogt-Koyanagi-Harada Syndrome. International Ophthalmology Clinics, 30, 279-285. https://doi.org/10.1097/00004397-199030040-00014

[39] Andreoli, M.T. and Andreoli, C.M. (2012) Surgical Rehabilitation of the Open Globe Injury Patient. American Journal of Ophthalmology, 153, 856-860.

https://doi.org/10.1016/j.ajo.2011.10.013 